\title{
Vorwort Heft 2-2011
}

\section{Hans-Christoph Grunau}

(C) Vieweg+Teubner und Deutsche Mathematiker-Vereinigung 2011

Am 26. Juni 2010 verstarb in Karlsruhe im Alter von 83 Jahren Wolfgang Walter, der das Gebiet der Differentialgleichungen in den letzten gut 50 Jahren mit zahlreichen wesentlichen Beiträgen bereichert hat. Von unnatürlichen Trennungslinien wie etwa der zwischen ,gewöhnlichen“ und ,,partiellen“ Differentialgleichungen hielt er nicht viel; in beiden Bereichen war er gleichermaßen heimisch und hat großen Gewinn aus deren Wechselspiel gezogen und fasziniert die sich entwickelnden Möglichkeiten computergestützten Beweisens verfolgt. In seinem Arbeitsbereich hat er die Bedeutung von Ungleichungen immer wieder hervorgehoben, deren konsequente Untersuchung und Verwendung maßgeblich zu den großen Fortschritten in der nichtlinearen Theorie von Differentialgleichungen beigetragen hat. Über dieses Gebiet hinausgehend hat er als Mitorganisator der „General Inequalities“-Tagungsserie Ungleichungen als verbindendes methodisches Element verschiedener mathematischer Teildisziplinen in den Vordergrund gestellt. Weithin bekannt ist Wolfgang Walter auch als Autor einer Reihe von Lehrbüchern, in denen man für grundlegende und weiterführende Vorlesungen gleichermaßen wahre Schätze an eleganten Beweisen und Herleitungen findet. Den vorliegenden Nachruf hat Wolfgang Reichel verfasst, der 1996 bei Wolfgang Walter promoviert hat und ein sehr lebendiges Bild von dessen Persönlichkeit entstehen lässt.

Wilhelm Stannat präsentiert in seinem Übersichtsartikel einige neuere Entwicklungen im Bereich der partiellen stochastischen Differentialgleichungen. Nach einer ausführlichen Einführung und der Darstellung einer Reihe physikalischer Beispiele setzt er den Schwerpunkt dabei auf die Untersuchung der Übergangshalbgruppen

H.-Ch. Grunau (凶)

Institut für Analysis und Numerik, Fakultät für Mathematik, Otto-von-Guericke-Universität, Postfach 4120, 39016, Magdeburg, Deutschland

e-mail: hans-christoph.grunau@ovgu.de 
der Lösungen solcher Gleichungen. In diesem Zusammenhang stellen sich die Fragen nach der Existenz einer invarianten Verteilung sowie nach der möglichen Beschreibung und Eindeutigkeit des zugehörigen Kolmogorov-Operators. Der Artikel präsentiert hier eine Vielzahl neuerer Resultate und Kriterien, mit denen sich diese und verwandte Fragen in vielen Situationen beantworten lassen.

Die Buchbesprechungen spannen in diesem Heft einen weiten inhaltlichen Bogen und behandeln Neuerscheinungen zur mathematischen Theorie der Supraleitung, zur Numerik nichtlokaler Operatoren und zur algebraischen Geometrie in Kodierungstheorie und Kryptographie.

Seit einigen Monaten ist auf den DMV-Webseiten auch ein Leserforum zum Jahresbericht installiert. Wie Sie dort sehen können, ist es nun für DMV-Mitglieder ziemlich einfach geworden, sich zum Jahresbericht zu äußern, sich an Diskussionen zu beteiligen oder neue Diskussionen zu beginnen. Auf Ihre Beiträge sind die Herausgeber unverändert sehr gespannt! 